\title{
FAMILIES OF VALUATIONS AND SEMIGROUPS OF FRACTIONARY IDEAL CLASSES
}

BY

\author{
ELBERT M. PIRTLE, JR.
}

Introduction. Let $R$ be an integral domain with quotient field $K$. For any valuation $v$ on $K$ which is nonnegative on $R$, we let $P(v)=\{x \in R \mid v(x)>0\} . P(v)$ is a prime ideal of $R$ and is called the center of $v$ on $R$. In this paper we are concerned mainly with integral domains $R$ which satisfy the following: There exists a family $F$ of valuations on $K$ such that

(i) Each $v \in F$ has rank one.

(ii) $R=\bigcap_{v \in F} R_{v}$.

(iii) $R_{v}=R_{P(v)}$, for each $v \in F$.

A family $F$ of valuations on $K$ is said to be of finite character if for $x \in K, x \neq 0$, there are only a finite number of $v \in F$ such that $v(x) \neq 0$. $R$ is called a Krull domain if there is a family $F$ of finite character satisfying (i), (ii), (iii), with the additional requirement that each $v \in F$ be discrete. $R$ is called an almost-Krull (AK) domain [7] if $R_{P}$ is a Krull domain for every proper nonzero prime $P$ of $R$. It follows that $R$ is almost Dedekind (AD) iff $R$ is an AK-domain in which proper prime ideals are maximal [7].

Using the family $F$ of valuations we construct a partially ordered semigroup $\mathscr{A}(R)$ of fractionary ideal classes in $\S 1$ and study the relation between $\mathscr{A}(R)$ and $\mathscr{D}(R)$, the divisor group of $R$ (see [1]). Necessary and sufficient conditions for $\mathscr{A}(R)$ and $\mathscr{D}(R)$ to be isomorphic are determined. In $\S 2$, condition $(S)$ of [3] is studied. $\S 3$ consists of an example.

The notation concerning $\mathscr{D}(R)$ is that of [1]. Otherwise, the notation of [8] is used. Prime ideals are always nonzero and not all of $R$.

1. In order to make this paper as self contained as possible we first list the necessary background results from [1]. $R$ will denote a commutative integral domain with identity and quotient field $K . I(R)$ will denote the collection of nonzero fractionary ideals of $R$. A fractionary ideal of the form $R x, x \in K, x \neq 0$, is called a principal fractionary ideal.

A relation $\prec$ is defined on $I(R)$ as follows: $A \prec B$ iff every principal fractionary ideal of $R$ which contains $A$ also contains $B$. The relation $\prec$ is a preorder on $I(R)$; i.e., $\prec$ is a symmetric, transitive relation. If we define $\equiv$ on $I(R)$ by $A \equiv B$ iff $A \prec B$ and $B<A$, then $\equiv$ is an equivalence relation on $I(R)$. For $A \in I(R), \operatorname{div}_{R}(A)$

Received by the editors January 8, 1969. 
denotes the equivalence class of $A$ with respect to $\equiv$ and is called the divisor of $A$; $\mathscr{D}(R)$ denotes the set of all such equivalence classes.

For $A \in I(R)$, we put $\tilde{A}=\bigcap_{A \subseteq R x} R x$. A fractionary ideal $B$ of $R$ is said to be divisoriel if $B=\widetilde{B}$. It follows that for $A \in I(R), \operatorname{div}_{R}(A)=\operatorname{div}_{R}(\tilde{A})$ and that $\tilde{A}$ is the unique divisoriel fractionary ideal belonging to $\operatorname{div}_{R}(A)$. It also follows from the definition that $(\tilde{A} \widetilde{B})^{\sim}=(A B)^{\sim}$ for $A, B \in I(R)$ so that $\mathscr{D}(R)$ together with the operation + , defined by $\operatorname{div}_{R}(A)+\operatorname{div}_{R}(B)=\operatorname{div}_{R}(A B)$, is a commutative semigroup with identity $0=\operatorname{div}_{R}(R)$. If we define $\leqq$ on $\mathscr{D}(R)$ by $\operatorname{div}_{R}(A) \leqq \operatorname{div}_{R}(B)$ iff $A \prec B$ then $\mathscr{D}(R)$ is a lattice ordered semigroup with respect to the partial ordering $\leqq$. Furthermore, $\mathscr{D}(R)$ is a group iff $R$ is completely integrally closed $[1$, p. 5 , Theorem 1].

Let $F$ be a family of valuations on $K$ with the following properties:

(i) Each $v \in F$ has rank one.

(ii) $R=\bigcap_{v \in F} R_{v}$.

(iii) For each $v \in F, R_{v}=R_{P(v)}$, where $P(v)$ denotes the center of $v$ on $R$.

Occasionally in place of (i) we shall substitute

(i') Each $v \in F$ has rank one and is discrete.

Definition 1.1. For $v \in F, A \in I(R)$, put $v(A)=\inf \{v(a) \mid a \in A\}$.

LEMMA 1.2. If $A, B \in I(R), v \in F$, then $v(A B)=v(A)+v(B)$.

Proof. See [4, p. 712], Theorem 1, part (2).

Now, for $A, B \in I(R)$, define $A \sim B$ iff $v(A)=v(B)$ for all $v \in F$. Then $\sim$ is an equivalence relation on $I(R)$. For $A \in I(R)$ we let $[A]$ denote the equivalence class of $A$ with respect to $\sim$, and we let $\mathscr{A}(R)$ denote the set of all such equivalence classes.

Define + on $\mathscr{A}(R)$ by $[A]+[B]=[A B]$. Then + is well defined. Since multiplication of fractionary ideals is commutative and associative, $\mathscr{A}(R)$ together with + is a commutative semigroup with identity $0=[R]$.

LEMMA 1.3. If $A=R x$ is a principal fractionary ideal, then $v(A)=v(x)$ for all $v \in F$.

Proof. $v(A)=v(R x)=\inf _{r x \in R x} v(r x)=\inf _{r \in R} v(r)+v(x)=v(1)+v(x)=v(x)$.

If $G$ is a group and $I$ is any nonempty index set, we let $G^{I}$ denote the direct product of $I$ copies of $G$ and we let $G^{(I)}$ denote the direct sum of $I$ copies of $G$. We shall assume that the value group of each $v \in F$ is a subgroup of the additive group of real numbers. When $v \in F$ is discrete we assume, without loss of generality, that the value group of $v$ is the additive group of integers. $X$ denotes the real numbers and $Z$ denotes the integers.

Proposition 1.4. Let $F=\left\{v_{i} \mid i \in I\right\}$ where $I$ is an index set. The map $f: \mathscr{A}(R) \rightarrow X^{I}$, defined by $f([A])=\left(v_{i}(A)\right)_{i \in I}$, is a monomorphism.

Proof. The proof is straightforward and is omitted. 
It follows from Proposition 1.4 that $\mathscr{A}(R)$ is a semigroup in which the cancellation law holds.

We now introduce a partial ordering for $\mathscr{A}(R)$.

Definition 1.5. For $[A],[B] \in \mathscr{A}(R)$, put $[A] \leqq[B]$ iff $v(A) \leqq v(B)$ for all $v \in F$.

PROPOSITION 1.6. $\mathscr{A}(R)$ is partially ordered by $\leqq$.

Proof. The proof is straightforward and is omitted.

As usual, if $[A],[B] \in \mathscr{A}(R)$ are such that $[A] \leqq[B]$ and $[A] \neq[B]$, we write $[A]<[B]$. Since $[R]=0 \in \mathscr{A}(R),[A] \in \mathscr{A}(R)$ is such that $[A] \geqq 0$ iff $A$ is an ideal of $R$. For if $A$ is an ideal of $R$, then $A \subseteq R$ so that $v(A) \geqq v(R)=0$, for all $v \in F$. On the other hand, if $[A] \geqq 0$, then $v(A) \geqq 0$ for all $v \in F$ so that $A \subseteq \bigcap_{v \in F} R_{v}=R$. Furthermore, if each $v \in F$ is discrete, then $[A]>0$ iff $A \subseteq P(v)$ for some $v \in F$. For if $[A]>0$, then, since each $v$ is discrete, $v(A) \geqq 1>0$ for some $v \in F$. But then $A \subseteq P(v)$, and conversely. We can use these properties of $\leqq$ to characterize the positive elements of $\mathscr{A}(R)$ when the elements of $F$ are discrete.

For $n$ a positive integer and $P$ a minimal prime of $R$, put $P^{(n)}=P^{n} R_{P} \cap R$. We shall assume that $F$ satisfies (i'), (ii), (iii) in Propositions 1.7 and 1.8.

Proposition 1.7. If $P$ is a minimal prime of $R$ and $P=P(v)$ for some $v \in F$, then $P^{(n)}=\{x \in R \mid v(x) \geqq n\}$ for every positive integer $n$.

Proof. We have $P^{(n)}=P^{n} R_{P} \cap R=\left(P R_{P}\right)^{n} \cap R$. So if $x \in P^{(n)}$, then $x \in\left(P R_{P}\right)^{n}$ and so $v(x) \geqq n$; i.e., $P^{(n)} \subseteq\{x \in R \mid v(x) \geqq n\}$. On the other hand, if $x \in R$ is such that $v(x) \geqq n$, then $x \in P$ and hence $x \in P R_{P}$. Since $v(x) \geqq n$ we have $x \in\left(P R_{P}\right)^{n}$, and so $x \in P^{(n)}$; i.e., $\{x \mid x \in R, v(x) \geqq n\} \subseteq P^{(n)}$.

As is well known, $P^{(n)}$ is a $P$-primary ideal of $R$.

Proposition 1.8. Let $A$ be an ideal of $R$ such that $[A]>0$, and let

$$
J=\left\{j \in I \mid v_{j}(A)>0\right\} .
$$

Then $A \subseteq \bigcap_{j \in J} P_{j}^{\left(n_{j}\right)}$, and $[A]=\left[\bigcap_{j \in J} P_{j}^{\left(n_{j}\right)}\right]$, where for each $j \in J, P_{j}=P\left(v_{j}\right)$ and $n_{j}=v_{j}(A)$.

Proof. If $x \in A, j \in J$, then $v_{j}(x) \geqq v_{j}(A)=n_{j}$; i.e., $x \in P_{j}^{\left(n_{j}\right)}$ by Proposition 1.7. Thus for each $j \in J, A \subseteq P_{j}^{\left(n_{j}\right)}$; i.e., $A \subseteq \bigcap_{j \in J} P_{j}^{\left(n_{j}\right)}$; which proves the first assertion. Now let $k \in J$. Since $A \subseteq \bigcap_{j \in J} P_{j}^{\left(n_{j}\right)}$, we have $v_{k}(A) \geqq v_{k}\left(\bigcap_{j \in J} P_{j}^{\left(n_{j}\right)}\right)$. On the other hand, let $x \in \bigcap_{j \in J} P_{j}^{\left(n_{j}\right)}$. Then $x \in P_{k}^{\left(n_{k}\right)}$, and $v_{k}(x) \geqq n_{k}=v_{k}(A)$; i.e., $v_{k}\left(\bigcap_{j \in J} P_{j}^{\left(n_{j}\right)}\right)$ $\geqq v_{k}(A)$. So if $k \in J$, then $v_{k}(A)=v_{k}\left(\bigcap_{j \in J} P_{j}^{\left(n_{j}\right)}\right)$. If $k \in I-J$, then $0=v_{k}(A) \geqq$ $v_{k}\left(\bigcap_{j \in J} P_{j}^{\left(n_{j}\right)}\right) \geqq 0$; i.e., $v_{k}(A)=0=v_{k}\left(\bigcap_{j \in J} P_{j}^{\left(n_{j}\right)}\right)$ for all $k \in I-J$, Thus

$$
v_{i}(A)=v_{i}\left(\bigcap_{j \in J} P_{j}^{\left(n_{j}\right)}\right)
$$

for all $i \in I$; i.e., $[A]=\left[\bigcap_{j \in J} P_{j}^{\left(n_{j}\right)}\right]$.

It follows that $\bigcap_{j \in J} P_{j}^{\left(n_{j}\right)}$ is the largest ideal $B$ of $R$ such that $[A]=[B]$. 
We now drop the assumption that each $v \in F$ is discrete so that $F$ satisfies (i), (ii) and (iii). Property (i) says that $R_{v}$ is a rank one valuation ring and hence completely integrally closed for each $v \in F$. Property (ii) shows that $R$ is the intersection of completely integrally closed overrings and hence is completely integrally closed. So (i) and (ii) insure that $\mathscr{D}(R)$ is a group. We now study relations between the semigroup $\mathscr{A}(R)$ and the group $\mathscr{D}(R)$.

The next two propositions have been proved in [6] for the case when $F$ is the family of essential valuations of an AD-domain $R$.

Proposition 1.9. Let $A \in I(R)$. Then, considering $[A]$ and $\operatorname{div}_{R}(A)$ as subsets of $I(R),[A] \subseteq \operatorname{div}_{R}(A)$.

Proof. Let $B \in[A]$. Then $v(B)=v(A)$ for all $v \in F$. If $A \subseteq R x$, then $v(A) \geqq v(R x)$ $=v(x)$, and so $v(B) \geqq v(x)$ for all $v \in F$. If $b \in B$, then $v(b)-v(x) \geqq 0$ for all $v \in F$; i.e., $v(b / x) \geqq 0$ for all $v \in F$. Thus if $b \in B$ then $b / x \in \bigcap_{v \in F} R_{v}=R$, and $b \in R x$; i.e., $B \subseteq R x$. Similarly, if $B \subseteq R y$ then $A \subseteq R y$. In this case, $\tilde{A}=\bigcap_{A \subseteq R y} R y=\bigcap_{B \subseteq R x} R x$ $=\widetilde{B}$, and $\operatorname{div}_{R}(A)=\operatorname{div}_{R}(B)$. Hence $B \in \operatorname{div}_{R}(A)$.

Proposition 1.10. The map $g: \mathscr{A}(R) \rightarrow \mathscr{D}(R)$ defined by $g([A])=\operatorname{div}_{R}(A)$ is an order preserving homomorphism of the partially ordered semigroup $\mathscr{A}(R)$ onto the lattice ordered group $\mathscr{D}(R)$.

Proof. Proposition 1.9 shows that $g$ is well defined and onto. It follows directly that $g$ is a homomorphism. To see that $g$ preserves order, suppose $[A],[B] \in \mathscr{A}(R)$ with $[A] \leqq[B]$. If $A \subseteq R x$, it follows as in the proof of 1.9 that $B \subseteq R x$ so that $A \subseteq B$, and hence $\operatorname{div}_{R}(A) \leqq \operatorname{div}_{R}(B)$.

Now let $T$ be a domain such that $R \subseteq T \subseteq K$ and such that there is a subfamily $G$ of $F$ such that $T=\bigcap_{w \in G} R_{w}$. It is easy to show that $G$ is a family of valuations for $T$ satisfying (i), (ii), (iii).

Proposition 1.11. The map $\sigma: \mathscr{A}(R) \rightarrow \mathscr{A}(T)$, defined by $\sigma([A])=[A T]$, is an order preserving homomorphism of $\mathscr{A}(R)$ onto $\mathscr{A}(T)$.

Proof. Here, $\mathscr{A}(T)$ denotes the semigroup of fractionary ideal classes of $T$ formed with the family $G$.

It is clear that $\sigma$ is well defined. To see that $\sigma$ is onto, let $\mathscr{U}$ be any nonzero fractionary ideal of $T$. Then $\mathscr{U}=(1 / d) \mathscr{B}$, where $\mathscr{B}$ is an ideal of $T, d \in R, d \neq 0$. Put $A=(1 / d) B$, where $B=\mathscr{U} \cap R$. It can be shown that $v(B)=v(\mathscr{B})$ for all $v \in G$, and hence $\sigma([A])=[\mathscr{U}]$. It is straightforward to show that $\sigma$ is a homomorphism which preserves order.

COROLlaRY 1.12. If $T$ is as in 1.11 , then $\mathscr{D}(T)$ is a homomorphic image of $\mathscr{A}(R)$. 
Let $T$ be as in 1.11, and consider the following diagram:

Diagram 1.13.

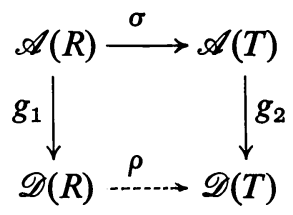

Here $\sigma$ is the homomorphism of $1.11, g_{1}$ and $g_{2}$ are the canonical homomorphisms of 1.10. In general, this diagram may not be completed commutatively by a homomorphism $\rho$. For let $R$ be an AD-domain which is not Dedekind, and let $F$ denote the family of essential valuations of $R$. By a result in [6], $R$ contains at least one proper prime $P$ which is not divisoriel. Then $P<\widetilde{P}$, and hence $\widetilde{P}=R$ since $P$ is maximal. Since $R$ is $\mathrm{AD}$, there is $v \in F$ such that $P=P(v)$, for some $v \in F$. Take $T=R_{P}=R_{v}$ and assume that $\rho$ completes the following diagram commutatively:

Diagram 1.14.

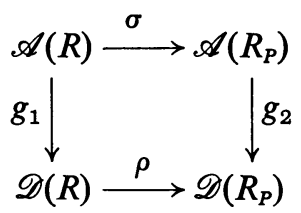

Then we must have $\rho\left(g_{1}([P])\right)=g_{2}(\sigma([P]))$. However, $g_{1}([P])=\operatorname{div}_{R}(P)=0$ (since $\tilde{P}=R)$ so that $\rho\left(g_{1}([P])\right)=0$; and on the other hand $\sigma([P])=\left[P R_{P}\right]$. But since $R_{P}$ is a Dedekind domain with unique maximal ideal $P R_{P}$, we have that $\operatorname{div}_{R_{P}}\left(P R_{P}\right)>0$; i.e., $g_{2}(\sigma([P]))=\operatorname{div}_{R_{P}}\left(P R_{P}\right)>0$. Thus $\rho g_{1} \neq g_{2} \sigma$, contradicting our assumption on $\rho$. This proves the assertion that, in general, Diagram 1.13 may not be completed commutatively.

Equivalent conditions for an AD-domain $R$ to be Dedekind are given in terms of $\mathscr{A}(R)$ in [6]. If we are to extend these results we need to know something about the inverses of elements of $\mathscr{A}(R)$ whenever they exist.

Proposition 1.15. If $[A] \in \mathscr{A}(R)$ has an inverse then $-[A]=[R: A]$.

Proof. Suppose $[A] \in \mathscr{A}(R)$ has an inverse $[B]$. Since the canonical map $g$ : $\mathscr{A}(R) \rightarrow \mathscr{D}(R)$ is a homomorphism, we must have that $g(-[A])=-g([A])=$ $-\operatorname{div}_{R}(A)=\operatorname{div}_{R}(R: A)$. Thus $g([B])=g(-[A])=\operatorname{div}_{R}(R: A)$. But by definition of $g, g([B])=\operatorname{div}_{R}(B)$ so that $\operatorname{div}_{R}(B)=\operatorname{div}_{R}(R: A)$. Since $R: A$ is divisoriel we have $B \subseteq \widetilde{B}=R: A=R: \tilde{A}$. Then $A B \subseteq A(R: A) \subseteq R$ so that $0=[A B] \geqq[A(R: A)] \geqq 0$. Thus $0=[A]+[B]=[A]+[R: A]$; i.e., $-[A]=[B]=[R: A]$.

Now consider the following diagram. 
Diagram 1.16.

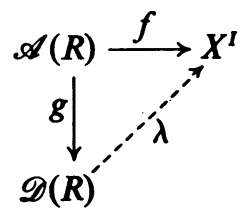

Here $g$ is the canonical homomorphism and $f$ is the homomorphism of $1.4 . g$ is surjective and $f$ is injective.

PROPOSITION 1.17. Diagram 1.16 may be completed commutatively by a homomorphism $\lambda$ iff $g$ is an isomorphism.

We can now prove the following theorem.

TheOREM 1.18. Let $R$ be an integral domain with quotient field $K$, and let $F$ be a family of valuations satisfying (i), (ii), (iii). The following statements are equivalent.

(1) $\mathscr{A}(R)$ is a group.

(2) $R: A=R: B \Rightarrow[A]=[B]$, for all $A, B \in I(R)$.

(3) $v(A)=v(\tilde{A})$ for all $A \in I(R)$ and $v \in F$.

(4) The map $g: \mathscr{A}(R) \rightarrow \mathscr{D}(R)$ is an isomorphism.

Proof. (1) $\Rightarrow$ (2) Suppose $\mathscr{A}(R)$ is a group. Let $A, B \in I(R)$ be such that $R: A$ $=R: B$. By 1.15 we have $-[A]=[R: A]=[R: B]=-[B]$ and hence $[A]=[B]$.

(2) $\Rightarrow$ (3) We have $R: A=R: \tilde{A}$ for all $A \in I(R)$. If (2) holds then $[A]=[\tilde{A}]$ for all $A \in I(R)$; i.e., $v(A)=v(\tilde{A})$ for all $v \in F, A \in I(R)$.

(3) $\Rightarrow$ (4) Consider Diagram 1.16. If $v(A)=v(\tilde{A})$ for all $v \in F$ and $A \in I(R)$, we can define $\lambda: \mathscr{D}(R) \rightarrow X^{I}$ by $\lambda\left(\operatorname{div}_{R}(A)\right)=\left(v_{i}(A)\right)_{i \in I}$. It follows that $\lambda$ is a homomorphism and that $\lambda \circ g=f$. By 1.17, $g$ is an isomorphism.

(4) $\Rightarrow$ (1) obvious.

We observe that the converse of statement (2) in 1.18 is always true in $R$. For if $[A]=[B]$, then $A \in[B] \subseteq \operatorname{div}_{R}(B)$, and $B \in \operatorname{div}_{R}(B)$ so that $\operatorname{div}_{R}(A)=\operatorname{div}_{R}(B)$ and hence $R: A=R: B$.

When the valuations in $F$ are discrete we obtain a partial generalization of a result in [6] with the aid of the following lemma.

LEMMA 1.19. Assume that each $v \in F$ is discrete. Then for each $v \in F$, if $\operatorname{div}_{R}(P(v))$ $\neq 0$ then $P(v)=(P(v))$.

Proof. We have $P(v) \subseteq(P(v))^{\sim} \subseteq R$. If $P(v)<(P(v))^{\sim}$, there is $x \in(P(v))^{\sim}, x \notin P(v)$. Then $v(x)=0=v(P(v)) \sim$. Also, for $w \in F, w \neq v$, we have $0=w(P(v)) \geqq w(P(v)) \sim 0$. Thus $\left[(P(v))^{\sim}\right]=0$. Since the canonical map $g$ from $\mathscr{A}(R)$ onto $\mathscr{D}(R)$ is a homomorphism, we should have $0=\left[(P(v))^{\sim}\right] \rightarrow \operatorname{div}_{R}(P(v))^{\sim}=\operatorname{div}_{R}(P(v))=0$. But $\operatorname{div}_{R}(P(v)) \neq 0$ by assumption. Thus we must have $(P(v))^{\sim}=P(v)$ if $\operatorname{div}_{R}(P(v)) \neq 0$.

THEOREM 1.20. Assume each $v \in F$ is discrete. Then the canonical map $g$ from $\mathscr{A}(R)$ onto $\mathscr{D}(R)$ is an isomorphism iff $P(v)$ is divisoriel for each $v \in F$. 
Proof. $(\Rightarrow)$ Suppose $g$ is an isomorphism. If $P=P(v)$ for some $v \in F$, then $[P]>0$ since $v(P)=1$. If $P$ is not divisoriel then $g([P])=\operatorname{div}_{R}(P)=0$, by Lemma 1.19. But then $g$ is not $1-1$ and hence not an isomorphism. For $[R]=0 \neq[P]$.

$(\Leftarrow)$ Suppose $P(v)$ is divisoriel for each $v \in F$. Then if $A \in I(R)$ is such that $\operatorname{div}_{R}(A)=0$ we must have $A \subseteq R$ (for this result see [1, bottom of p. 4]). Moreover, $A \nsubseteq P(v)$ for any $v \in F$. For if $A \subseteq P=P(v)$ for some $v \in F$, then $\operatorname{div}_{R}(A) \geqq \operatorname{div}_{R}(P)$ $>0$, a contradiction. Thus $g([A])=0$ iff $[A]=0$. Now suppose $[A],[B] \in \mathscr{A}(R)$ are such that $g([A])=g([B])$. Then $\operatorname{div}_{R}(A)=\operatorname{div}_{R}(B)$ so that $\operatorname{div}_{R}(A)-\operatorname{div}_{R}(B)=0$ $=\operatorname{div}_{R}(B)-\operatorname{div}_{R}(A)$; i.e., $\operatorname{div}_{R}(A: B)=0=\operatorname{div}_{R}(B: A)$. Since $g([A: B])=g([B: A])$ $=0$, we must have $[A: B]=0=[B: A]$. Since each $v \in F$ is discrete, for each $v \in F$ there is $x \in A: B$ such that $v(x)=v(A: B)=0$. Now $x B \subseteq A$ (by definition of $A: B$ ) so that $v(x)+v(B)=v(x B) \geqq v(A)$; i.e., $v(B) \geqq v(A)$. Thus $v(B) \geqq v(A)$ for all $v \in F$. Similarly $v(A) \geqq v(B)$ for all $v \in F$, and $[A]=[B]$. This shows that $g$ is $1-1$ and hence an isomorphism.

When $R$ is $\mathrm{AD}$, the author has shown in [6] that $P(v)$ is divisoriel for each $v \in F$ iff $R$ is Dedekind. To date, however, the author has been unable to prove the following conjecture: If $R$ is $\mathrm{AK}$ and $P(v)$ is divisoriel for each $v \in F$, then $R$ is a Krull domain.

When $R$ is $\mathrm{AK}$, we do have the following theorem.

THEOREM 1.21. Let $R$ be an AK-domain with family $F$ of essential valuations and let $\Delta$ denote the collection of maximal ideals of $R$. Every minimal prime of $R$ is divisoriel iff $\tilde{A}=\bigcap_{M \in \Delta}\left(A R_{M}\right)^{\sim}$ for every ideal $A$ of $R$.

Proof. Here $\tilde{A}=\bigcap_{A \subseteq R x} R x$ and $\left(A R_{M}\right)^{\sim}=\bigcap_{A R_{M} \subseteq R_{M} y} R_{M} y$. For any maximal ideal $M$ of $R, F_{M}$ denotes the family of essential valuations of the Krull domain $R_{M}$. Recall that $F_{M} \subseteq F$.

$(\Rightarrow)$ Let $A$ be an ideal of $R$. If $M$ is any maximal ideal of $R$ then $v(A)=v\left(A R_{M}\right)$ for all $v \in F_{M}$. Since $R_{M}$ is a Krull domain, $v\left(A R_{M}\right)=v\left(A R_{M}\right) \sim$ for all $v \in F_{M}$ so that $v(A)=V\left(A R_{M}\right) \sim$ for all $v \in F_{M}$.

Case 1. $v(A)=0$ for all $v \in F$.

Then $P<A$ for every minimal prime $P$ of $R$. In this case $\tilde{A}=R=\bigcap_{M \in \Delta} R_{M}=$ $\bigcap_{M \in \Delta}\left(A R_{M}\right)^{\sim}$.

Case 2. $v(A)>0$ for some $v \in F$.

For each maximal ideal $M$ of $R$, if there is $v \in F_{M}$ such that $0<v(A)=v\left(A R_{M}\right)^{\sim}$, then we can write

$$
\left(A R_{M}\right)^{\sim}=\bigcap_{v_{i} \in F_{M} ; v_{i}(A)>0} Q_{i}^{\left(n_{i}\right)},
$$

where $n_{i}=v_{i}\left(A R_{M}\right) \sim$ and $Q_{i}$ is the center of $v_{i}$ on $R_{M}$. Then for each $i$ such that $v_{i}(A)>0$ we have $Q_{i}=P_{i} R_{i}$ where $P_{i}=P\left(v_{i}\right)$ in $R$. Thus $\left(A R_{M}\right)^{\sim}=\bigcap_{i}\left(P_{i} R_{M}\right)^{\left(n_{i}\right)}$ $=\bigcap_{i}\left(\left(P_{i} R_{M}\right)^{n_{i}} R_{P_{i}}\right) \cap R_{M}=\bigcap_{i}\left(P_{i}^{n_{i}} R_{P_{i}} \cap R_{M}\right)$ where $i$ runs over all indices such that $v_{i} \in F_{M}$ and $v_{i}(A)>0$, and $n_{i}=v_{i}(A)$ for each such $i$. It can then be shown that, $C=\bigcap_{M \in \Delta}\left(A R_{M}\right)^{\sim}=\bigcap\left\{P_{i}^{\left(n_{i}\right)} \mid v_{i} \in F, v_{i}(A)>0\right\}$. Then $[C]=[A]$. Since $[A]=\operatorname{div}_{R}(A)$, it follows that $\tilde{A}=C$. 
$(\Leftarrow)$ Suppose $P$ is a minimal prime of $R$. If $M \in \Delta$, then either $P \subseteq M$ or $P \nsubseteq M$. If $P \nsubseteq M$ then $P R_{M}=R_{M}$. If $P \subseteq M$, then $P R_{M}$ is a minimal prime of the Krull domain $R_{M}$ and thus $\left(P R_{M}\right)^{\sim}=P R_{M}$. Since $P$ is contained in some maximal ideal $M$ we have $\tilde{P}=\bigcap_{M \in \Delta}\left(P R_{M}\right)^{\sim}=\bigcap_{M \in \Delta} P R_{M}=P$.

We now drop the assumption that $R$ is an AK-domain and assume only that $F$ satisfies axioms (i), (ii), (iii) at the beginning of this section. The next lemma tells us more about the elements of $\mathscr{A}(R)$ which have inverses and enables us to partially describe $\mathscr{D}(R)$ in certain cases where $\mathscr{A}(R)$ may not be a group.

LemMA 1.22. If $[A] \in \mathscr{A}(R)$ is such that $[A]$ has an inverse then $[A]=[\tilde{A}]$.

Proof. If $[A] \in \mathscr{A}(R)$ has an inverse then $-[A]=[R: A]$ by Proposition 1.15. Now $A \subseteq \tilde{A}$ and $R: A=R: \tilde{A}$. Thus $A(R: A) \subseteq \tilde{A}(R: A)=\tilde{A}(R: \tilde{A}) \subseteq R$. These containment relations yield the following: $0=[A(R: A)] \geqq[\tilde{A}(R: \tilde{A})] \geqq 0$. Thus

$$
0=[A]+[R: A]=[\tilde{A}]+[R: A] \text { and }[A]=[\tilde{A}] \text {. }
$$

CoROLlaRY 1.23. If $[A],[B]$ have inverses in $\mathscr{A}(R)$, then $[\tilde{A}]+[\tilde{B}]=[\tilde{A} \widetilde{B}]=[A B]^{\sim}$; i.e., $v(\tilde{A} \widetilde{B})=v(A B)^{\sim}$ for all $v \in F$.

Proof. By 1.22 above, if $[A],[B]$ have inverses then $[A]=[\tilde{A}]$ and $[B]=[\tilde{B}]$. Moreover, $[A]+[B]$ has an inverse. Thus $[A]+[B]=[A B]=[A B]^{\sim}$ by 1.22 ; i.e., $[\tilde{A}]+[\widetilde{B}]=[\tilde{A} \widetilde{B}]=[A B]^{\sim}$.

Now consider the map $\rho: \mathscr{D}(R) \rightarrow X^{I}$ defined by $\rho\left(\operatorname{div}_{R}(A)\right)=\left(v_{i}(\tilde{A})\right)_{i \in I} \cdot \rho$ is well defined, for if $\operatorname{div}_{R}(A)=\operatorname{div}_{R}(B)$ then $\tilde{A}=\widetilde{B}$. Conversely, if $\left(v_{i}(\tilde{A})\right)_{i \in I}=\left(v_{i}(\widetilde{B})\right)_{i \in I}$ then $[\tilde{B}]=[\tilde{A}]$ and so $\operatorname{div}_{R}(A)=\operatorname{div}_{R}(\tilde{A})=\operatorname{div}_{R}(\widetilde{B})=\operatorname{div}_{R}(B)$ by the remark following the proof of 1.18 . Thus $\rho$ is $1-1$. We can now give a description of $\mathscr{D}(R)$ when $R$ is fairly well behaved.

THeOREM 1.24. The map $\rho: \mathscr{D}(R) \rightarrow X^{I}$ defined by $\rho\left(\operatorname{div}_{R}(A)\right)=\left(v_{i}(\tilde{A})\right)_{i \in I}$ is 1-1. Furthermore $\rho$ is a homomorphism iff $[\tilde{A}] \in \mathscr{A}(R)$ has an inverse for all $A \in I(R)$.

Proof. The first assertion is proved in the immediately preceding remarks. We now prove the second assertion.

$(\Rightarrow)$ Suppose $\rho$ is a homomorphism. Then since $\mathscr{D}(R)$ is a group, for $\operatorname{div}_{R}(A)$ $\in \mathscr{D}(R), \quad-\operatorname{div}_{R}(A)=\operatorname{div}_{R}(R: A)$. Thus $\rho\left(\operatorname{div}_{R}(A)+\operatorname{div}_{R}(R: A)\right)=0=\rho\left(\operatorname{div}_{R}(A)\right)$ $+\rho\left(\operatorname{div}_{R}(R: A)\right)=\left(v_{i}(\tilde{A})\right)_{i \in I}+\left(v_{i}(R: A)\right)_{i \in I}$. It follows that $[\tilde{A}]$ has an inverse in $\mathscr{A}(R)$.

$\Leftrightarrow$ Suppose that $[\tilde{A}]$ has an inverse for all $A \in I(R)$. By Corollary 1.23, we have that $[\tilde{A} \widetilde{B}]=[A B]^{\sim}$ for all $A, B \in I(R)$. Thus for $A, B \in I(R)$ we have $\rho\left(\operatorname{div}_{R}(A B)\right)$ $=\left(v_{i}(A B)\right)_{i \in I}=\left(v_{i}(\tilde{A} \tilde{B})\right)_{i \in I}=\left(v_{i}(\tilde{A})\right)_{i \in I}+\left(v_{i}(\tilde{B})\right)_{i \in I}=\rho\left(\operatorname{div}_{R}(A)\right)+\rho\left(\operatorname{div}_{R}(B)\right)$ and $\rho$ is a homomorphism.

Now, let $R$ be an AK-domain. Then $R_{P}$ is a Krull domain for any prime ideal $P$ of $R$. However, these are not the only Krull domains $T$ such that $R \subseteq T \subseteq K$. For if $\Delta=\left\{P_{1}, \ldots, P_{n}\right\}$ is any finite collection of prime ideals of $R$ then $T=\bigcap_{P_{l} \in \Delta} R_{P_{i}}$ is also a Krull domain. Thus there is a large class of Krull domains $T$ such that 
$R \subseteq T \subseteq K$. When $R$ is an AK-domain in which every minimal prime is divisoriel we always have that $\mathscr{D}(T)$ is a homomorphic image of $\mathscr{D}(R)$, where $T$ is an AKdomain such that $R \subseteq T \subseteq K$. For, $\mathscr{A}(T)$ is a homomorphic image of the group $\mathscr{A}(R)$ and so is a group. Then $\mathscr{A}(R) \cong \mathscr{D}(R)$ and $\mathscr{A}(T) \cong \mathscr{D}(T)$. When $T$ is a Krull domain and $R$ is an AK-domain for which the map $\rho$ of Theorem 1.24 is a homomorphism we also get that $\mathscr{D}(T)$ is a homomorphic image of $\mathscr{D}(R)$ as follows.

Proposition 1.25. Let $R$ be an AK-domain and let $T$ be a Krull domain such that $R \subseteq T \subseteq K$. If $[\tilde{A}]$ has an inverse for every $[A] \in \mathscr{A}(R)$ then the map $\tau: \mathscr{D}(R)$ $\rightarrow \mathscr{D}(T)$, defined by $\tau\left(\operatorname{div}_{R}(A)\right)=\operatorname{div}_{T}(\tilde{A} T)$, is a homomorphism of $\mathscr{D}(R)$ onto $\mathscr{D}(T)$.

Proof. $\tau$ is well defined, for if $\operatorname{div}_{R}(A)=\operatorname{div}_{R}(B)$ then $\tilde{A}=\tilde{B}$ so that $\tilde{A} T=\tilde{B} T$. Now consider the following diagram.

Diagram 1.26.

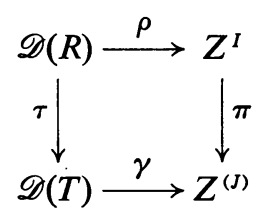

Here, $I$ is the index set for the family of essential valuations of $R$; $J$ is the index set for the family of essential valuations of $T ; \pi$ is the projection of $Z^{I}$ onto $Z^{(J)}$; $\rho$ is the (injective) homomorphism of $1.24 ; \gamma$ is the injection of 1.4. It is well known that $\gamma$ is also surjective; i.e., $\gamma$ is an isomorphism. Consider the map $\gamma^{-1} \circ \pi \circ \rho$ : $\mathscr{D}(R) \rightarrow \mathscr{D}(T)$. We have, for $\operatorname{div}_{R}(A) \in \mathscr{D}(R),\left(\gamma^{-1} \circ \pi \circ \rho\right)\left(\operatorname{div}_{R}(A)\right)=\left(\gamma^{-1} \circ \pi\right)$ $\times\left(v_{i}(\tilde{A})\right)_{i \in I}=\gamma^{-1}\left(\left(v_{j}(\tilde{A})\right)_{j \in J}\right)$. Since $T$ is a Krull domain we have that $v(B)=v(\widetilde{B})$ for all fractionary ideals $B$ of $T$ and all essential valuations $v$. Thus $\left(v_{j}(\tilde{A})\right)_{j \in J}$ $=\left(v_{j}(\tilde{A} T)\right)_{j \in J}=\left(v_{j}(\tilde{A} T)^{\sim}\right)_{j \in J}$ so that $\gamma^{-1}\left(\left(v_{j}(\tilde{A})\right)_{j \in J}\right)=\operatorname{div}_{R}(\tilde{A} T)$. Then $\gamma^{-1} \circ \pi \circ \rho=\tau$ and $\tau$ is a homomorphism since $\tau$ is a composition of homomorphisms. To see that $\tau$ is surjective it is sufficient to show that for every divisoriel fractionary ideal $\mathscr{U}$ of $T$ there is a divisoriel fractionary ideal $A$ of $R$ such that $\tau\left(\operatorname{div}_{R}(A)\right)=\operatorname{div}_{T}(\mathscr{U})$. So let $\mathscr{U}$ be a fractionary ideal of $T$. There are elements $x, y \in K$ such that $\mathscr{U}=T x$ $\cap T y$ [1, p. 13]. Let $A=R x \cap R y$. Then $A$ is divisoriel and $v_{j}(A)=v_{j}(\mathscr{U})$ for all $j \in J$ and $\tau\left(\operatorname{div}_{R}(A)\right)=\operatorname{div}_{T}(\mathscr{U})$.

2. Let $R$ be an integral domain with quotient field $K$. Suppose that $F$ is a family of valuations on $K$ satisfying the following:

(1) $R=\bigcap_{v \in F} R_{v}$,

(2) $R_{v}=R_{P(v)}$, for each $v \in F$.

Following Gilmer in [3], we make the following definition.

Definition 2.1. We say that $R$ satisfies property ( with respect to $F$ iff for distinct subsets $F_{1}, F_{2}$ of $F$ we have that $\bigcap_{w \in F_{1}} R_{w} \neq \bigcap_{u \in F_{2}} R_{u}$.

When $R$ is a Prufer domain and $F$ is the family of valuations induced by the collection of maximal ideals, then property (*) is the same as property (*) in [2]. 
For $v \in F$, we let $F_{v}=F-\{v\}$.

Proposition 2.2. $R$ has property ( ) with respect to $F$ iff for each $v \in F$, $\bigcap_{w \in F_{v}} R_{w} \nsubseteq R_{v}$.

Proof. The proof is substantially the same as that of Lemma 1 in [2] and is omitted.

COROLlaRY 2.3. If $R$ satisfies (*) with respect to $F$ and if $G$ is any nonempty subset of $F$, then $T=\bigcap_{u \in G} R_{u}$ satisfies (*) with respect to $G$.

We note that if $R$ satisfies (*) with respect to $F$ then $P(v) \nsubseteq P(w)$ for $v \neq w$. For if $P(v) \subseteq P(w)$ for some $w \neq v$, then $R_{P(w)} \subseteq R_{P(v)}$; i.e., $R_{w} \subseteq R_{v}$. Then we have the following: $\left(\bigcap_{u \in F-\{v, w\}} R_{u}\right) \cap\left(R_{v} \cap R_{w}\right)=\left(\bigcap_{u \in F-\{v, w\}} R_{u}\right) \cap R_{w}=\bigcap_{u \in F_{v}} R_{u}$, and $F \neq F_{v}$, a contradiction.

Proposition 2.4. If $F$ is of finite character and is such that $P(u) \nsubseteq P(v)$ if $u \neq v$, then $R$ satisfies ( with respect to $F$.

Proof. Let $v \in F$ and let $x \in R, x \neq 0$, be such that $v(x)>0$. Let $v_{1}, \ldots, v_{n}$ be the distinct (from $v$ and each other) valuations such that $v_{i}(x) \neq 0, i=1, \ldots, n$. There exists $y \in\left(\bigcap_{i=1}^{n} P\left(v_{i}\right)\right)-P(v)$. For if $\bigcap_{i=1}^{n} P\left(v_{i}\right) \subseteq P(v)$, then $\prod_{i=1}^{n} P\left(v_{i}\right) \subseteq \bigcap_{i=1}^{n} P\left(v_{i}\right)$ $\subseteq P(v)$ and so $P\left(v_{i}\right) \subseteq P(v)$ for some $j, 1 \leqq j \leqq n$, contradicting our hypothesis. Choose $n$ large enough so that $w\left(y^{m} / x\right) \geqq 0$ for $w \in F_{v}$. This is possible since $F$ is of finite character and $w(y) \geqq 0$ for all $w \in F_{v}$. Then $w\left(y^{m} / x\right) \geqq 0$ for all $w \in F_{v}$ and $v\left(y^{m} / x\right)$ $=-v(x)<0$. Thus $y^{m} / x \in\left(\bigcap_{w \in F_{v}} R_{w}\right)-R_{v}$; i.e., $\bigcap_{w \in F_{v}} R_{w} \nsubseteq R_{v}$. So $R$ satisfies ( with respect to $F$ by 2.2 .

Let $R$ be an integral domain with family $F$ of valuations satisfying (1) and (2) listed at the beginning of this section. $R$ is called a generalized Krull domain if $F$ satisfies the following two additional properties (see [5]).

(3) Each $v \in F$ has rank one.

(4) $F$ is of finite character.

COROLlaRY 2.5. If $R$ is a Krull domain, or a generalized Krull domain with family $F$ of valuations, then $R$ satisfies (*) with respect to $F$.

Proof. In this case $F$ is a family of rank one valuations of finite character, so that if $u, v \in F, u \neq v$, then $P(v) \nsubseteq P(u)$.

Proposition 2.6. Let $R$ be an $\mathrm{AD}$-domain. The following conditions on $R$ are equivalent.

(1) $R$ satisfies (*) with respect to $F$, the family of essential valuations of $R$.

(2) $R$ is Dedekind.

(3) Every minimal prime of $R$ is divisoriel.

Proof. (1) $\Leftrightarrow(2)$ is Theorem 3 of [3].

$(2) \Leftrightarrow(3)$ is found in [6]. 
Thus we see that in the case of almost-Dedekind domains, the divisoriel property of the minimal prime ideals completely determines whether or not $R$ satisfies property (*). We shall see that the divisoriel property of the minimal primes is always sufficient for $R$ to satisfy (*).

Proposition 2.7. Let $R$ be an integral domain with family $F$ of valuations such that

(i) Each $v \in F$ has rank one.

(ii) $R=\bigcap_{v \in F} R_{v}$.

(iii) $R_{v}=R_{P(v)}$ for each $v \in F$.

If $P(v)$ is divisoriel for each $v \in F$, then $R$ satisfies (ख) with respect to $F$.

Proof. We note that since $R$ is the intersection of rank one valuation rings, $R$ is completely integrally closed and hence $\mathscr{D}(R)$ is a group. If each $P(v)$ is divisoriel, then each $v \in F$ is discrete. For if $P=P(v)$ is divisoriel we must have $P^{2}<P$. For if $P^{2}=P$, then $\operatorname{div}_{R}\left(P^{2}\right)=\operatorname{div}_{R}(P)$; i.e., $2 \operatorname{div}(P)=\operatorname{div}(P)$. Thus $\operatorname{div}(P)=0$ and $\widetilde{P}=R \neq P$, contradicting $\widetilde{P}=P$.

Since $P^{2}<P$, we have $P^{2} R_{P}<P R_{P}$ and so $R_{P}$ is a discrete valuation ring. We now show that $\{P(v) \mid v \in F\}$ is the set of all minimal divisoriel primes of $R$. Clearly, $\{P(v) \mid v \in F\}$ is contained in the set of all divisoriel minimal primes. Now let $P$ be a minimal, divisoriel prime of $R$. If $P \neq P(v)$ for any $v \in F$, then $P \nsubseteq P(v)$ for any $v \in F$ and so $v(P)=0$ for all $v \in F$; i.e., $[P]=0$. But then we would have $g([P])=0$; i.e., $\operatorname{div}(P)=0$; i.e., $\widetilde{P}=R$, contradicting $\widetilde{P}=P<R$. So we must have that

$$
\{P(v) \mid v \in F\}
$$

is the set of all divisoriel minimal primes of $R$. Now let $G$ be any subset of $F$ such that $R=\bigcap_{u \in G} R_{u} . P(u)$ is divisoriel for each $u \in G$ since $G \subseteq F$. By what we have just shown, $\{P(u) \mid u \in G\}$ is the collection of all minimal divisoriel primes of $R$; i.e., $G=F$. Thus for any $v \in F, \bigcap_{u \in F_{v}} R_{u} \nsubseteq R_{v}$ and so $R$ satisfies (*) with respect to $F$.

The first part of the proof of Proposition 2.7 shows that if $P$ is the center of a rank one valuation $v$, then $v$ is discrete if $P$ is divisoriel. This enables us to characterize Krull domains in the class of generalized Krull domains as follows.

COROLlaRY 2.8. Let $R$ be a generalized Krull domain with family $F$ of valuations. $R$ is a Krull domain iff $P(v)$ is divisoriel for each $v \in F$.

Let $R$ be an integral domain with quotient field $K$ and let $F$ be a family of valuations on $K$ satisfying conditions (1) and (2) stated at the beginning of this section. Let $x$ be an indeterminate and let $F^{\prime}$ denote the family of valuations on $K(x)$ which are canonical extensions of elements of $F$. Let $G$ denote the family of $p(x)$-adic valuations on $K(x)$, where $p(x)$ is a nonconstant irreducible polynomial in $K[x]$. Then $F^{\prime} \cup G$ is a family of valuations on $K(x)$ satisfying (1) and (2) with $R[x]$ in place of $R$. 
Proposition 2.9. If $R$ satisfies ( $)$ with respect to $F$, then $R[x]$ satisfies (*) with respect to $F^{\prime} \cup G$.

Proof. Let $w \in F^{\prime} \cup G$. If $w \in G$, then $w$ is a $p(x)$-adic valuation for some nonconstant irreducible polynomial $p(x) \in K[x]$. Without loss of generality we may assume that $p(x) \in R[x]$. Suppose $p(x)=a_{n} x^{n}+a_{n-1} x^{n-1}+\cdots+a_{1} x+a_{0}, a_{i} \in R$. Let $b=\prod_{a_{k} \neq 0} a_{k}$. Then $b \neq 0$ since $a_{n} \neq 0$, and $v(b)=\sum_{a_{k} \neq 0} v\left(a_{k}\right) \geqq \min _{0 \leqq j \leqq n} v\left(a_{j}\right)$ for all $v \in F$ since $b \in R$ and $a_{k} \in R$ for all $k=0,1, \ldots, n$, and every $v \in F$ is nonnegative on $R$. Then for $v^{\prime} \in F^{\prime}, v^{\prime}(b / p(x))=v^{\prime}(b)-v^{\prime}(p(x))=v(b)-\min _{0 \leqq j \leqq n} v\left(a_{j}\right)$ $\geqq 0$. If $u \in G$ and $u \neq w$, then $u$ is a $q(x)$-adic valuation for some nonconstant irreducible polynomial $q(x)$ such that $q(x) \nmid p(x)$. Then $u(b / p(x))=0$. Thus $b / p(x)$ $\in \bigcap_{u \in(F \cup G)_{w}}(R[x])_{u} . \quad b / p(x) \notin(R[x])_{w}$ since $w(b / p(x))=-1<0$. Thus if $w \in G$ $\bigcap_{u \in\left(F^{\prime} \cup G\right)_{w}}(R[x])_{u} \nsubseteq(R[x])_{w}$. On the other hand, if $w \in F^{\prime}$, then $w=v^{\prime}$ for some $v \in F$. Since $\bigcap_{u \in F_{v}} R_{u} \nsubseteq R_{v}$, there is $a \in\left(\bigcap_{u \in F_{v}} R_{u}\right)-R_{v} \subseteq\left(\bigcap_{u^{\prime} \in F^{\prime} v^{\prime}}, R[x]_{u^{\prime}}\right) \cap K[x]$ $=\left(\bigcap_{u^{\prime} \in F^{\prime} v^{\prime}}, R[x]_{u^{\prime}}\right) \cap\left(\bigcap_{z \in G} R[x]_{z}\right)=\bigcap_{w \in\left(F^{\prime} \cup G\right)_{v^{\prime}}} R[x]_{w}$, and $a \notin R_{v}$. Then $a \notin R[x]_{v^{\prime}}$, for $v^{\prime}(a)=v(a)<0$. Thus for every $w \in F^{\prime} \cup G$ we have $\bigcap_{u \in\left(F^{\prime} \cup G\right)_{w}} R[x]_{u} \nsubseteq R[x]_{w}$ and thus $R[x]$ satisfies ( with respect to $F^{\prime} \cup G$ by 2.2 .

3. In [6] it was shown that if $R$ is an almost-Dedekind domain with family $F$ of essential valuations, then $R$ is Dedekind iff every minimal prime of $R$ is divisoriel. Thus in an AD-domain $R$, every minimal prime is divisoriel iff $F$ is of finite character. In $\S 1$ it was conjectured that if $R$ is an AK-domain with family $F$ of essential valuations, then $R$ is Krull if $P(v)$ is divisoriel for each $v \in F$; i.e., $F$ is of finite character if $P(v)$ is divisoriel for each $v \in F$. In this section we give an example to show that this conjecture is false if the $\mathrm{AK}$ requirement is dropped. We also give an example of an AK-domain which is neither a Krull domain nor an AD-domain.

Let $R$ denote the set of entire functions, $C$ denote the set of complex numbers, $Z$ denote the additive group of integers. It is well known that $R$ is an integral domain under the usual pointwise definitions of addition and multiplication. For $a \in C$ we define $v_{a}: R-\{0\} \rightarrow Z$ by $v_{a}(f(z))=n$ if $a$ is a zero of $f(z)$ of order $n$. If $a$ is not a zero of $f(z)$ then $v_{a}(f(z))=0$. If $f(z) \equiv 0$ we put $v_{a}(f(z))=+\infty$ for each $z \in C$. It is easy to show that each $v_{a}$ can be extended to a valuation on the quotient field of $R$. We let $F$ denote this family of valuations. $F$ has the following properties: (i) Each $v \in F$ has rank one and is discrete; (ii) $R=\bigcap\left\{R_{v} \mid v \in F\right\}$; (iii) $R_{v}=R_{P(v)}$ for each $v \in F$; (iv) For $a \in C, P\left(v_{a}\right)=(z-a) R$, and hence is divisoriel; (v) $F$ is not of finite character. Furthermore, $P(v)$ is maximal for each $v \in F$. However, these are not all the maximal ideals of $R$. For let $\left\{z_{n}\right\}_{n=1}^{\infty}$ be a sequence of complex numbers such that $\lim z_{n}=\infty$. For each positive integer $m$, let $f_{m}(z)$ be an entire function whose zeros are exactly $\left\{z_{m}, z_{m+1}, \ldots\right\}$. The ideal generated by $\left\{f_{1}(z)\right.$, $\left.f_{2}(z), \ldots\right\}$ is proper and is contained in a maximal ideal $M$. However, $R_{M}$ is not a Krull domain. It follows that $R$ is not AK.

It was shown in [7] that if $R$ is $\mathrm{AK}$ and $X_{1}, \ldots, X_{n}$ are indeterminates, then $R\left[X_{1}, \ldots, X_{n}\right]$ is AK. Let $R$ be an AD-domain which is not a Dedekind domain. 
Such a domain is given in example 2 of [2]. Then $R\left[X_{1}, \ldots, X_{n}\right]$ is an AK-domain which is neither a Krull domain nor an AD-domain. We observe that example 1 of [2] is a generalized Krull domain which is not a Krull domain.

Author's Note. This paper constitutes a part of a Ph.D. dissertation written under the direction of Professor Paul J. McCarthy at the University of Kansas. The author is indebted to Professor McCarthy for his most valuable counsel and advice during the course of this work.

\section{REFERENCES}

1. N. Bourbaki, Algèbre commutative, Chapitre 7, Hermann, Paris, 1965.

2. R. W. Gilmer, Jr., Overrings of Prufer domains, J. Algebra 4 (1966), 331-340.

3. R. W. Gilmer, Jr. and William Heinzer, Irredundant intersections of valuation rings, Math. Z. 103 (1968), 306-317.

4. Malcom Griffin, Some results on v-multiplication rings, Canad. J. Math. 19 (1967), 710-722.

5. J. L. Mott, On the complete integral closure of domains of Krull type, Math. Ann. 173 (1967), 234-240.

6. Elbert M. Pirtle, Jr., A note on almost Dedekind domains (to appear).

7. _- Integral domains which are almost Krull, J. Sci. Hiroshima Univ. Ser. A-I Math. 32 (1968), 101-107.

8. O. Zariski and P. Samuel, Commutative algebra, Vol. II, Van Nostrand, Princeton, N. J., 1961 .

The UNIVERSITY OF MisSOURI,

Kansas City, Missouri 